\title{
Financial Considerations of Hospital-Based Palliative Care
}

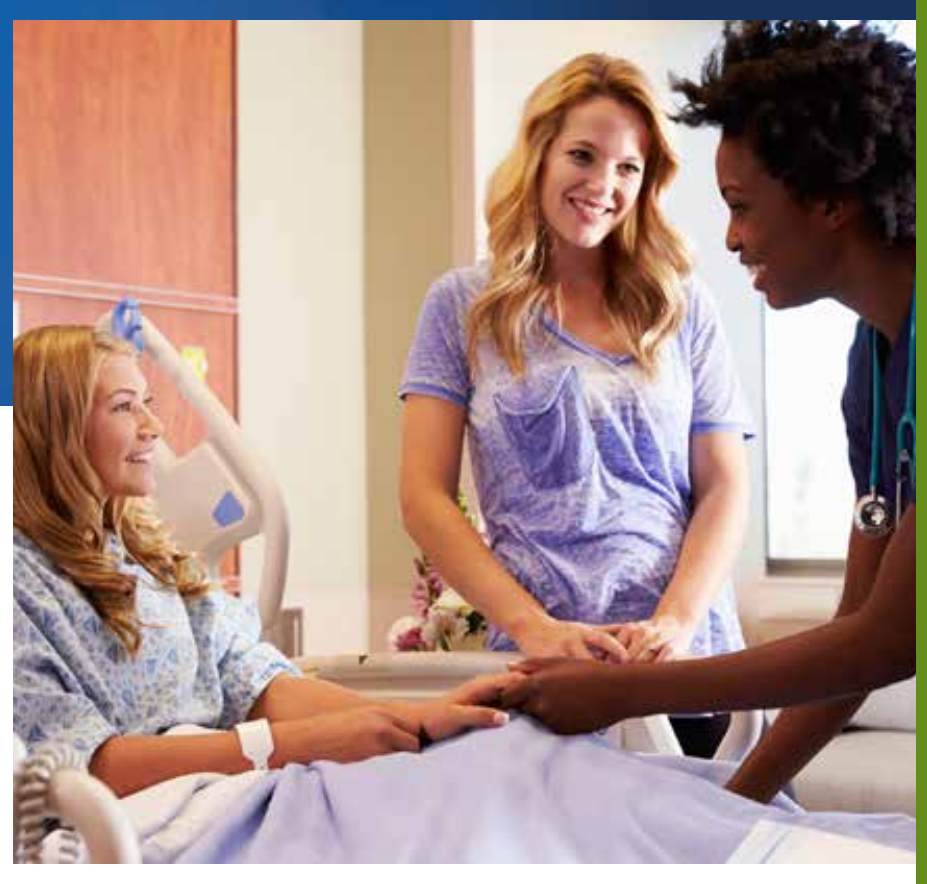

Renee T. Sullender and Sarah A. Selenich 
RTI Press publication RR-0027-1603

This PDF document was made available from www.rti.org as a public service of RTI International. More information about RTI Press can be found at http://www.rti.org/rtipress.

RTI International is an independent, nonprofit research organization dedicated to improving the human condition by turning knowledge into practice. The RTI Press mission is to disseminate information about RTI research, analytic tools, and technical expertise to a national and international audience. RTI Press publications are peerreviewed by at least two independent substantive experts and one or more Press editors.

\section{Suggested Citation}

Sullender, R. T., and Selenich, S. A. (2016). Financial Considerations of Hospital-Based Palliative Care. RTI Press Publication No. RR-0027-1603. Research Triangle Park, NC: RTI Press. http://dx.doi.org/10.3768/rtipress.2016.rr.0027.1603

This publication is part of the RTI Press Research Reports series.

RTI International

3040 East Cornwallis Road

PO Box 12194

Research Triangle Park, NC

27709-2194 USA

Tel: +1.919.541.6000

E-mail: rtipress@rti.org

Website: www.rti.org
O2016 RTI International. All rights reserved. Credit must be provided to the author and source of the document when the content is quoted. No part of this document may be reproduced without permission in writing from the publisher. RTI International is a registered trademark and a trade name of Research Triangle Institute.

http://dx.doi.org/10.3768/rtipress.2016.rr.0027.1603 www.rti.org/rtipress 


\section{Contents}

About the Authors

Acknowledgments ii

Abstract ii

Introduction

Methods 1

Results 2

The Impact of Hospital-Based Palliative Care on Hospital Costs and Health Care Spending 2

Hospital-Based Palliative Care Reimbursement 3

Discussion 4

Financial Research Considerations 4

Implementation Research Considerations 5

Policy Considerations 6

$\begin{array}{ll}\text { Limitations } & 7\end{array}$

$\begin{array}{ll}\text { Conclusion } & 7\end{array}$

References 1

\section{About the Authors}

At the time of writing, Renee $\mathrm{T}$. Sullender, BA, was a public health analyst in RTI International's Social Policy, Health, and Economics Research Unit in Research Triangle Park, North Carolina. She is now a medical student at the University of Wisconsin School of Medicine and Public Health in Madison, Wisconsin. At the time of writing, Sarah A. Selenich, MPP, was a health policy analyst in RTI's Social Policy, Health, and Economics Research Unit in Washington, DC. She is now a senior associate at the Moran Company in Arlington, Virginia. 


\section{Acknowledgments}

This research was supported by internal RTI funds. The authors gratefully acknowledge Kathleen N. Lohr (PhD, MPhil, MA), Nathan D. West (MPA), Nicole Coomer (PhD), Franziska S. Rokoske (MS, PT), and M. Alexis Kirk (BA) for their review and suggestions.

\section{Abstract}

Palliative care is an interdisciplinary care philosophy addressing patient and family needs and goals without providing a cure for the underlying disease. Palliative care can be successfully provided alongside curative care, which does focus on treating the disease. Studies have indicated that palliative care offers a variety of quality of life benefits to both the patient and family. Prompted by rapid growth of hospital-based palliative care, we explored the literature to better understand the financial incentives and barriers to these programs. Although patients who receive palliative care in the hospital have lower hospital costs than matched patients who do not receive palliative care, many hospitals face challenges in being reimbursed for services rendered by their interdisciplinary teams. In some cases, hospitals may absorb 50 percent of the costs of their palliative care teams because of lack of adequate reimbursement. Despite the opportunity for cost savings for a variety of stakeholders, without payment reform hospitals may be constrained from providing palliative care to all who might benefit. Additional research is needed to understand how patients, hospitals, and payers may participate in cost savings attributable to palliative care so that policymakers can effectively promote these services. 


\section{Introduction}

Palliative care is a comprehensive philosophy addressing physical, psychosocial, and spiritual needs for the patient and family. Unlike most hospice care, which is limited to those with a prognosis of 6 months if their terminal illness runs a normal course, palliative care is available at any stage of an illness alongside curative care. Palliative care may ease symptoms and burdens and address patient and family needs, but traditionally it has not been used to directly treat the patient's disease. Alternatively, curative care treats existing medical conditions; for instance, a patient may receive chemotherapy in an attempt to cure cancer. Patients may elect to receive palliative care alongside or without curative care.

Members of an interdisciplinary palliative care team might include clinicians, social workers, chaplains, therapists, and psychologists; they work together to coordinate care and provide comfort and aid. Ongoing communication among the palliative care team, medical specialists, and the patient and family can optimize symptom management and supportive care in accordance with the patient's and family's care goals. For example, a patient with chronic obstructive pulmonary disorder (COPD) might receive treatment for shortness of breath and for coughing, anxiety, and depression in accordance with his or her needs and preferences. Palliative care services also address psychosocial and other care needs for the patient and family. Patients can receive palliative services in a variety of settings, such as at home, nursing facilities, and hospitals.

Research has proven that palliative care provides numerous benefits to both patients and their families and caregivers. A study of newly diagnosed metastatic non-small-cell lung cancer patients, for instance, showed that those receiving palliative care had better quality of life, were less likely to have depressive symptoms, and survived longer despite receiving less aggressive care at the end of life, compared with those not receiving palliative care (Temel et al., 2010). A second study found that Medicaid palliative care recipients in hospitals spent less time in the intensive care unit (ICU), were less likely to die in the ICU, and were more likely to elect to enter hospice to receive care at home, compared with a matched group of patients not receiving palliative care (Morrison et al., 2011). Wright and colleagues (2010) found that patients with cancer who died in the ICU or hospital without palliative care experienced more physical and emotional distress and had worse quality of life at the end of life than patients who died at home with hospice-provided palliative care. Additionally, compared with home-based hospice deaths, ICU deaths are associated with greater risk for posttraumatic stress disorder and more psychiatric illness in bereaved caregivers.

In this research report, we are particularly interested in the growing role of palliative care in hospitals and the financial implications of the increase. Palliative care programs in hospitals with at least 50 beds grew 157.1 percent between 2001 and 2011; in 2015, 67 percent of hospitals with 50 or more beds had palliative care teams, an increase from 63 percent in 2011 (Center to Advance Palliative Care, 2013; Morrison \& Meier, 2011, 2015). We explored reasons for expansion of palliative care teams in hospitals, identifying studies indicating the hospital cost-reducing and quality-improving capabilities of palliative care. By 2040, the number of people in the US over the age of 65 will make up more than 20 percent of the population, and the number of people with chronic conditions is quickly growing (Administration on Aging, 2016). Given the range of documented benefits of quality of care, our hypothesis is that hospital-based palliative care services may help alleviate the financial burden of caring for an aging and ill US population.

\section{Methods}

This research on hospital-based palliative care was borne out of an initial literature review to provide a broader context of issues related to hospice care. Hospice is included in the palliative care umbrella but has important differences; the most important distinction is that patients receiving hospice care do not continue to receive curative treatment for the terminal diagnosis. By contrast, patients receiving palliative care outside of hospice can choose to receive curative care for all illnesses. During our 
hospice research, palliative care financing emerged as a topic worth further investigation. The wealth of literature on the positive outcomes experienced by patients receiving palliative care convinced us of the importance of examining financial struggles and the benefits of hospital-based palliative care programs.

Although literature exists on hospital costs for patients receiving palliative care, the growth in hospital-based palliative care seemed to signal a need for reexamination, especially given the complexity of reimbursement systems. Our approach involved understanding the financial incentives and barriers to the adoption of palliative care teams. Having identified hospital-based palliative care as a topic deserving additional research, we conducted literature searches related to palliative care financing and hospital-based palliative care reimbursement, focusing on material since 2000 . We used the University of North Carolina Online Library System, the National Library of Medicine MEDLINE database (via PubMed), Google Scholar, and Google search engines. Key search terms included palliative care reimbursement, hospital-based palliative care, hospital palliative care cost, palliative care payment, and palliative care financing.

\section{Results}

We divided results of the literature search into two sections: one focused on hospital-based palliative care costs and health care spending and the other addressing hospital-based palliative care reimbursement. It was difficult at times to find concrete information on reimbursement for hospitalbased palliative care, especially services rendered by non-medical professionals. We address additional gaps in the literature in the Discussion section as they pertain to future research questions.

\section{The Impact of Hospital-Based Palliative Care on Hospital Costs and Health Care Spending}

Health care spending is a topic of great interest in the United States. Reining in costs and improving outcomes while respecting patient preferences is challenging. Hospitals want to provide their patients with the best care, but they also face very real financial constraints; this is particularly true for institutions that serve rural and safety-net populations. In our literature review, we found a mixed picture about financial incentives and barriers to hospital-based palliative care. To further complicate the picture, some studies examine costs, and others examine charges.

Several studies suggest that the cost of providing the full range of services for a patient is lower when the patient receives palliative care than when the patient does not receive such services. In a study of four diverse urban New York State hospitals, researchers calculated that Medicaid patients receiving palliative care between 2004 and 2007 incurred on average $\$ 6,900$ less in hospital costs for a given admission than those who received usual care (Morrison et al., 2011). However, it is not entirely clear whether costs associated with palliative care are subtracted from that total. In the study, a patient was identified as receiving palliative care if the attending physician requested a palliative care consultation, the patient was seen and evaluated by the palliative care team at least once, and at least one set of recommendations was made by the palliative care team to the patient's primary team. Compared with a matched group of patients not receiving palliative care services, hospital patients receiving palliative care services incurred, on average, $\$ 4,098$ less in costs per admission for live discharges and $\$ 7,563$ less per admission for patients who died in the hospital. The average total cost per admission was $\$ 36,741$ for a patient discharged alive who received usual care and $\$ 32,643$ for a matched patient who also received palliative care. For just ICU costs, patients discharged alive who received palliative care incurred on average $\$ 2,678$ lower costs than those who did not receive palliative care.

In another study of data from 2002 to 2004 of eight geographically and structurally diverse hospitals, laboratory and ICU costs for patients over the age of 18 receiving palliative care who were discharged alive were significantly lower than the laboratory and ICU costs of matched patients not receiving palliative care (Morrison et al., 2008). The hospital costs of patients receiving palliative care who died in the hospital were lower in laboratory, ICU, and pharmacy fields. Patients discharged alive incurred \$1,696 less in costs per admission, and those who died incurred $\$ 4,908$ 
less in costs per admission compared with matched patients receiving usual care only. Investigators excluded patients with stays less than 7 and more than 30 days for two main reasons: the unlikelihood that short-stay patients would receive palliative care consultations and the difficulty of generalizing data on the long-stay patients.

Researchers have also found that cost reductions extend beyond the hospital stay. A three-site randomized trial of hospitalized patients enrolled in the study between June 2002 and December 2003 found that mean health costs in the 6 months postdischarge were $\$ 6,766$ lower per patient among those who had received hospital-based palliative care compared with usual care only (Gade et al., 2008). The primary driver was a reduction in readmission costs; while there was no difference in the number of hospital readmissions, patients receiving palliative care had significantly fewer ICU stays on readmission. Intervention patients readmitted to the hospital were again followed by the palliative care team.

Costs reflect all health services provided in the 6 months after discharge. With palliative care staffing costs of $\$ 1,911$ per patient, as determined by the time spent by each team member multiplied by their hourly wage and benefits, the net savings were $\$ 4,855$ per patient (Gade et al., 2008). The interdisciplinary inpatient palliative care consultative service consisted of a palliative care physician and nurse, hospital social worker, and chaplain. At all sites, the entire team met prior to every consultation, met with the patient and family, and then met again to develop a plan and organize follow-up. The team was available Monday through Friday, and a palliative care physician was on call after hours. Upon discharge, the team provided a palliative care discharge plan to the primary care physician. Study participants were limited to those 18 years of age and older who were hospitalized with at least one life-limiting diagnosis and whose attending physician noted he or she "would not be surprised if the patient died within 1 year."

In a study of a public hospital in Indiana in 2005, patients who received a palliative care consultation service incurred $\$ 6,907$ less in hospital charges than demographically and clinically similar patients who did not receive a consultation service (Bendaly,
Groves, Juliar, \& Gramelspacher, 2008). Note, however, that this particular study examined hospital charges rather than hospital costs, and that the study only included patients aged 50 or older who died during a hospitalization of at least 3 days.

Achieving meaningful cost reductions while promoting quality is clearly possible when hospitalized patients receive palliative care together with their standard treatment, which reduces costs by an average of $\$ 4,098$ per admission for patients discharged alive (Morrison et al., 2011). If just 2 percent to 6 percent of Medicaid patients discharged from a hospital received palliative care while in the hospital, annual Medicaid hospital spending in the State of New York could be reduced by $\$ 84$ million to $\$ 252$ million, respectively. For this particular calculation, the investigators assumed that every New York hospital with 150 beds or more had a fully operational palliative care team.

\section{Hospital-Based Palliative Care Reimbursement}

Although evidence suggests that further proliferation of hospital-based palliative care is desirable, financial hurdles can be substantial. Currently, no palliative care benefit or clearly defined method of reimbursement exists for hospital-based services. Coverage for palliative care varies widely. Moreover, identifying discrete services as being part of a comprehensive palliative care approach is difficult.

Under Medicare Part A, hospitals do not receive a payment adjustment for palliative care services. Clinicians can bill palliative care consultant services through Medicare Part B and some commercial insurance plans (Center to Advance Palliative Care, 2011; National Hospice and Palliative Care Organization, 2001). However, interdisciplinary team members such as chaplains, social workers, and bereavement counselors may not receive reimbursement from public or private payers for provision of services essential to the success and spirit of palliative care.

As a result of the lack of reimbursement from payers, hospitals typically provide at least half of the overall palliative care program funding (Center to Advance 
Palliative Care, 2011). A 2014 study of hospital-based palliative care programs in California found that the percentage of programs with chaplains decreased from 77 percent in 2007 to 57 percent in 2011, and the percentage of social workers decreased from 80 percent to 58 percent (Pantilat, O'Riordan, \& Bruno, 2014). The percentage of programs comprising clinicians from four or more disciplines also decreased from 61 percent in 2007 to 45 percent in 2011, perhaps reflecting the challenges of providing palliative care services for which the hospital receives no reimbursement.

Small, rural, and safety-net hospitals may face additional barriers in funding and staffing palliative care programs (Morrison \& Meier, 2011). These hospitals may be less likely than other hospitals to be part of a health system with sufficient resources to fund a palliative care team.

\section{Discussion}

\section{Financial Research Considerations}

The exact degree to which hospitals may benefit financially from implementation of a palliative care program is unclear. This outcome likely varies based on the quality and breadth of the programs and their patient and payer mix. What is evident is the need for additional research into how savings are allocated when the hospital costs associated with a patient who receives palliative care are lower than costs for a matched patient who does not receive palliative care. One key point is the mix of stakeholders who may benefit; these can include patients, hospitals, and payers, depending on the hospital and the type of insurance coverage, if any, the patient has.

Insofar as total hospital costs are lower because of palliative care, depending on the type of insurance the patient may have and the length of stay, the patient may save money. For instance, in 2015, Medicare beneficiaries had to pay a \$1,260 Part A hospital inpatient deductible for each benefit period (Centers for Medicare \& Medicaid Services [CMS], 2014). The benefit period begins the day a patient is admitted as an inpatient and ends when he or she has not received any inpatient hospital care for 60 days in a row. If the patient is readmitted after a benefit period ends, a new period begins, and the patient must pay the inpatient deductible for the new benefit period.

Patients are responsible for different coinsurance amounts depending on the length of the benefit period. For instance, Medicare patients pay no coinsurance during days 1-60 of the benefit period and pay $\$ 315$ in coinsurance per day between days 61 and 90. Therefore, a Medicare patient could save money if palliative care reduces his or her total hospital costs below the $\$ 1,260$ deductible threshold or if palliative care can reduce the length of stay past day 60. If palliative care can reduce the likelihood of hospital readmissions, Medicare patients could also be less likely to have to pay the inpatient deductible for a new benefit period.

Patients with private insurance are responsible, on average, for an 18 percent coinsurance rate for hospital admissions after meeting an average \$346 annual hospital deductible (Kaiser Family Foundation and Health Research \& Educational Trust, 2013). Table 1 illustrates how palliative care could reduce coinsurance costs for patients who have already met their deductible. The table utilizes the $\$ 4,908$ average reduction in costs per admission for patients who received palliative care alongside usual care and who died in the hospital (Morrison et al., 2008). This simple estimate suggests that families of patients who received palliative care and died in the hospital might save around $\$ 883$ for such a stay.

\section{Table 1. Hospital costs to patients with coinsurance who died in the hospital}

\section{Costs}

Patient A: Receives No Palliative Care $\$ 30,000$
Patient B: Receives Palliative Care $\$ 25,092^{\mathrm{a}}$

\begin{tabular}{lcc} 
Total Hospital Cost & $\$ 30,000$ & $\$ 25,092^{\mathrm{a}}$ \\
\hline Cost to Patient/Family with $18 \%$ coinsurance & $0.18(\$ 30,000)=\$ 5,400$ & $0.18(\$ 25,092)=\$ 4,517$ \\
\hline a Patients who received palliative care alongside usual care and who died in the hospital incurred an average $\$ 4,908$ less in hospital costs per admission than those
\end{tabular}
who received usual care only (Morrison et al., 2008). 
Determining whether hospitals save money depends on how they are reimbursed by payers. For instance, if total costs are lower because of reduced ICU stays and fewer unnecessary drugs, do hospitals share in any savings? If so, to what extent? Hospital expenses may be reduced if the hospital does not have to conduct as many tests and procedures. Reduced hospital length of stay for patients receiving palliative care could also lead to bed availability for other patients. However, hospital revenue may be lower if the case is consequently assigned a lower-weight diagnosisrelated group (DRG).

Research is needed to better understand the relationship between palliative care and reimbursement, considering such hospital variables as size, geography, patient population characteristics, ownership (such as for-profit or nonprofit), and type (such as an academic medical center, charity hospital, rural hospital, etc.). For instance, in the randomized control trial discussed previously that looked at post-discharge cost savings, the three participating hospitals were part of the same integrated delivery health plan, and the study was approved by that health plan (Gade et al., 2008). Policymakers need to consider how characteristics of hospitals could influence the potential for cost savings and how that may affect hospitals' decisions about implementing such programs.

Researchers also need to determine how the expense of maintaining the palliative care team itself compares with any savings experienced by the hospital. An important concern is that hospitals must often absorb a large percentage of the cost of the palliative care team because of low or no reimbursement for many of their services. Even if hospitals may reduce their cost of treating patients with time, without sufficient reimbursement, they may be unable to begin or continue providing palliative care, at least with the types of disciplines and services that are essential to the palliative care team composition.

Finally, with respect to payers, one would expect the federal Medicare program to save money because its payments to hospitals would be reduced. In particular, Medicare may be able to expect savings on patients who would have been high-cost outlier patients without the intervention of palliative care.

Private payers may save money depending on their arrangements with hospitals. For instance, if an insurer will pay only a fixed sum for a certain diagnosis or treatment, and if palliative care reduces total hospital costs, the cost reduction may not translate into savings for the payer. Alternatively, if the payer covers a certain percentage of hospital costs, and palliative care reduces hospital costs, then the payer could save money. Several research questions remain unanswered regarding whether different payers may share in savings from hospital-based palliative care, given the considerable number of possible variables involved. Payers may have both a quality and a financial incentive to promote palliative care based upon their arrangements with hospitals.

\section{Implementation Research Considerations}

Research on the relationship between the nature of the palliative care team and the quality of care they deliver, as well as the health and financial outcomes of that care, is needed to maximize the effectiveness of palliative care. Palliative care teams by nature are interdisciplinary, requiring experts from several fields to be truly successful. Such teams need to work well together, have experienced members, and be willing to coordinate and communicate with a variety of disciplines and providers. The interdisciplinary palliative care teams of the hospitals assessing Medicaid patients received training that was in accordance with the National Quality Forum's framework and preferred practices for palliative and hospice care quality (Morrison et al., 2011). Although not all palliative care teams may need such training, policymakers should know whether team members must have a certain level of expertise or training to be effective in improving quality of care and patients' health outcomes and satisfaction.

Additional research is needed on which hospitalized patients might benefit the most from palliative care. For instance, the Morrison and colleagues cost studies referenced earlier were limited to patients with diagnoses of severe and potentially lifethreatening ailments, such as cancer, HIV/AIDS, 
heart failure, and COPD; future studies should examine whether palliative care produces better quality and financial outcomes based on diagnosis and prognosis (Morrison et al., 2008; Morrison et al., 2011). Palliative care may have a more meaningful impact on patients with chronic pain and previously unmanageable symptoms than those with lower levels of symptoms. Based on patient exclusions in the aforementioned cost studies, future research goals should also include understanding the quality and financial benefit of palliative care for short- and longstay patients (fewer than 7 days and more than 30 days), as well as for pediatric palliative care patients.

\section{Policy Considerations}

Although some hospitals must absorb significant palliative care costs under current payment mechanisms, hospital-based programs have been growing. Given the established quality-of-life improvements for patients, however, payment reform is still needed to promote faster and more comprehensive proliferation of services. Restructuring palliative care reimbursement or including palliative care in a public sector benefit program should be considered to ensure that palliative care is readily accessible to all patients who might benefit from such services.

In 2014, the Institute of Medicine (IOM) recommended that government and private health insurers provide financial incentives for including palliative care for patients at the end of life, recognizing opportunities to improve quality and reduce costs (Committee on Approaching Death, 2014). As palliative care can be beneficial to patients and families at any point along the illness trajectory, the IOM recommendation may support movement toward comprehensive reimbursement for all palliative care activities. The creation of reimbursable billing codes, such as for pastoral visits, bereavement counseling, and discussions about treatment options, is one possible avenue for ensuring that palliative care is reimbursed and available for all patients who would benefit.

Experts have previously suggested that payers might not currently offer comprehensive palliative care coverage because they have no existing reimbursement mechanism upon which to model coverage (Lubell, 2010). The Center to Advance Palliative Care (CAPC), an organization at the Icahn School of Medicine at Mount Sinai (New York City) dedicated to increasing the availability of palliative care, has recognized the development of reimbursable billing codes as a best practice of payment innovation (Warner \& Gualtieri-Reed, 2014).

Policymakers could encourage payers and hospitals to improve identification and differentiation of palliative care practices through billing codes. In July 2015, the Center for Medicare \& Medicaid Services (CMS) proposed paying physicians for optional end-of-life discussions with Medicare patients by activating two CPT billing codes (Lowes, 2015). However, CMS did not set a rate for the codes, so Medicare administrative contractors, who are normally private health insurers, will generally be responsible for the decision to reimburse physicians when processing Medicare claims. While these discussions are advance-care planning specific for patients at the end of life, we believe this remains positive movement toward reimbursement for additional care planning discussions.

Expanding the existing Medicare hospice benefits to allow Medicare patients who are not terminally ill to receive palliative services without forgoing curative care is another potential route to consider. Twenty percent of Medicare beneficiaries have at least five chronic conditions, and two-thirds of Medicare spending covers these beneficiaries' care (Belluck, 2014). These types of patients in particular, despite not necessarily being terminally ill, could benefit from the improved oversight and care coordination that are expected to accompany palliative care.

The Medicare Care Choices Model demonstration may provide valuable insights into the joint provision of palliative and curative care. In this program, Medicare and dually eligible (Medicare and Medicaid) beneficiaries can receive care from hospice providers while concurrently receiving curative treatment, not necessarily while in the hospital. Hospices will receive $\$ 400$ per beneficiary per month to provide services. The demonstration is restricted to patients with certain diagnoses (e.g., COPD, advanced cancer, HIV/ AIDS), although patients with other illnesses may 
also benefit from palliative services. Furthermore, the model is restricted to patients who meet hospice eligibility requirements, not patients at any stage of their illness. Depending on the quality and financial outcomes of the demonstration, the evaluation will inform the costs and benefits of reimbursing palliative care and reimbursement strategies. Due to robust interest, CMS has already expanded the model from an originally anticipated 30 Medicare-certified hospices to over 140 hospices and extended the model from 3 to 5 years, targeting around 150,000 Medicare beneficiaries. The first phase of the model began in January 2016, and the second phase of participating hospices will begin in January 2018 (CMS, 2015).

An increase in palliative care might be a natural outgrowth of new payment and delivery systems. One example is accountable care organizations (ACOs) that are participating in CMS initiatives that seek to achieve cost savings and improve their performance in areas such as readmissions, care coordination, and concordance with patient preferences. As previously noted, additional research is needed on the distribution of any savings or costs among stakeholders, but ACOs with a financial incentive to reduce costs while maintaining quality care may be in a stronger position than traditional health systems to accommodate any reduced revenue.

The prevalence of palliative care teams in hospitals across the country is not uniform. The CAPC found that while 90 percent of hospitals with at least 300 beds had a palliative care team in 2015, only 29 percent of hospitals with fewer than 50 beds had teams (Morrison \& Meier, 2015). Interestingly, forprofit hospitals of any size are less likely to have a palliative care team than nonprofit hospitals. The CAPC has noted the reasons for this discrepancy are unknown. Perhaps for-profit hospitals are more hesitant than nonprofits to absorb costs for the palliative care team despite potential future savings.

Geography also plays an important role in determining accessibility of palliative care to patients and in distributing potential savings. In 2011, in the Northeast, 73 percent of hospitals with 50 or more beds had palliative care teams, as compared with 51 percent in the South (Morrison \& Meier, 2011).
Policymakers may wish to consider existing barriers when crafting policy to promote palliative care, as lack of reimbursement may constrain smaller and rural hospitals in particular from providing services to all who might benefit.

\section{Limitations}

A limitation of our literature review is that it did not meet the standard requirements for a formal systematic review, as doing so was not our purpose as part of a larger background report providing context about hospice care. We believe that we identified the most pertinent and recent scientific literature and research organization reports on palliative care financing. Although literature exists on the possibility of cost savings from hospital-based palliative care, a substantial number of research questions remain outstanding, largely regarding which stakeholders may benefit from savings under different financial reimbursement arrangements among patients, payers, and hospitals. In-depth research on how the hospital costs of palliative care teams compare with hospital savings must be undertaken in order to inform any policy decisions and business cases. We also note that costs and savings as discussed here do not include the monetized value of life quality or quantity or utilize quality-adjusted life years (QALYs), and thus may not include the full impact of palliative care interventions. As other researchers have found, this type of costeffectiveness is challenging and may lead to studies assessing costs separately from outcomes (Smith, Brick, O’Hara, \& Normand, 2014).

\section{Conclusion}

We live in a nation in which people are living longer while also suffering from a variety of diseases. As many of these and other patients would benefit from palliative care, even while simultaneously receiving treatment, a system-wide effort is needed to expand these types of services. The current approach-in which hospitals fund much of their own palliative care programs-is insufficient for providing services to all who might benefit given the potential cost savings involved. Improving existing hospitalbased services and expanding new programs are both possible if patients, hospitals, and payers can 
better understand the quality and financial benefits involved. Additional research is needed to understand which stakeholders save money under different financial arrangements, as well as the characteristics of effective palliative care teams. Only with such information can hospital administrators, clinicians, and policymakers enact effective policies encouraging appropriate palliative care. As the US population ages and the burden of treating the seriously ill grows, promoting palliative care as a way of both ensuring quality and reducing health care costs is increasingly important.

\section{References}

Administration on Aging. (2016). Aging statistics. Retrieved January 29, 2016, from http://www.aoa.acl. gov/Aging_Statistics/index.aspx

Belluck, P. (2014, August 30). Coverage for end-oflife talks gaining ground. The New York Times. Retrieved January 29, 2016, from http://www.nytimes. com/2014/08/31/health/end-of-life-talks-may-finallyovercome-politics.html

Bendaly, E. A., Groves, J., Juliar, B., \& Gramelspacher, G. P. (2008). Financial impact of palliative care consultation in a public hospital. Journal of Palliative Medicine, 11(10), 1304-1308.

Center to Advance Palliative Care. (2011). The case for hospital palliative care. Retrieved February 5, 2016, from https://media.capc.org/filer public/06/90/069053fe-12bf-4485-b973-d290f7c2ecbf/ thecaseforhospitalpalliativecare_capc.pdf

Center to Advance Palliative Care. (2013). Growth of palliative care in US hospitals: 2013 snapshot. Retrieved February 4, 2016, from https://media.capc. org/filer_public/0d/db/0ddbecbc-8dc7-449d-aa50584960f18880/capc-growth-analysis-snapshot-2013.pdf

Centers for Medicare \& Medicaid Services. (2014). Medicare costs at a glance: 2015. Retrieved February 5, 2016, from https://www.cms.gov/Outreach-andEducation/Medicare-Learning-Network-MLN/ MLNProducts/Downloads/Costs-At-Glance-FactSheet-ICN909005.pdf

Centers for Medicare \& Medicaid Services. (2015, July 20). Medicare Care Choices Model - frequently asked questions. Retrieved January 29, 2016, from http:// innovation.cms.gov/initiatives/Medicare-CareChoices/faq.html

Committee on Approaching Death: Addressing Key Endof-Life Issues. (2014). Dying in America: Improving quality and honoring individual preferences near the end of life. Washington, DC: Committee on Approaching Death: Addressing Key End-of-Life Issues.
Gade, G., Venohr, I., Conner, D., McGrady, K., Beane, J., Richardson, R. H., . . . Della Penna, R. (2008). Impact of an inpatient palliative care team: A randomized control trial. Journal of Palliative Medicine, 11(2), 180-190.

Kaiser Family Foundation and Health Research \& Educational Trust. (2013). Employer health benefits: 2013 annual survey. Menlo Park, CA: Kaiser Family Foundation and Chicago, IL: Health Research \& Educational Trust. Retrieved January 29, 2016, from http://kaiserfamilyfoundation.files. wordpress.com/2013/08/8465-employer-healthbenefits-20132.pdf

Lowes, R. (2015, July 8). CMS proposes paying for endof-life discussions. Medscape Medical News. Retrieved January 29, 2016, from http://www.medscape.com/ viewarticle/847689

Lubell, J. (2010). Easing their pain: Palliative care grows despite reimbursement issues. Modern Healthcare. Retrieved January 29, 2016, from http:// www.modernhealthcare.com/article/20100531/ MAGAZINE/100529913

Morrison, R. S., Dietrich, J., Ladwig, S., Quill, T., Sacco, J., Tangeman, J., \& Meier, D. E. (2011). Palliative care consultation teams cut hospital costs for Medicaid beneficiaries. Health Affairs, 30(3), 454-463. http:// dx.doi.org/10.1377/hlthaff.2010.0929

Morrison, R. S., \& Meier, D. E. (2011). Key findings: $A$ state-by-state report card on access to palliative care in our nation's hospitals. New York: Center to Advance Palliative Care.

Morrison, R. S, \& Meier, D. E. (2015). America's care of serious illness: 2015 state-by-state report card on access to palliative care in our nation's hospitals. Retrieved February 5, 2016, from Center to Advance Palliative Care website, https://reportcard.capc.org/wp-content/ uploads/2015/08/CAPC-Report-Card-2015.pdf 
Morrison, R. S., Penrod, J. D., Cassel, J. B., CaustEllenbogen, M., Litke, A., Spragens, L., \& Meier, D. E. (2008). Cost savings associated with US hospitals palliative care consultation programs. Archives of Internal Medicine, 168(16), 1783-1790.

National Hospice and Palliative Care Organization. (2001). Hospital-hospice partnerships in palliative care: Creating a continuum of service. Alexandria, VA: National Hospice and Palliative Care Organization. Retrieved January 29, 2016, from http://www.nhpco.org/sites/ default/files/public/NHPCO-CAPCreport.pdf

Pantilat, S. Z., O’Riordan, D. L., \& Bruno, K. A. (2014). Two steps forward, one step back: Changes in palliative care consultation services in California hospitals from 2007 to 2011. Journal of Palliative Medicine, 17(11), 1214-1220.

Smith, S., Brick, A., O’Hara, S., \& Normand, C. (2014). Evidence on the cost and cost-effectiveness of palliative care: A literature review. Palliative Medicine, 28(2), 130-150. http://dx.doi.org/10.1177/0269216313493466
Temel, J. S., Greer, J. A., Muzikansky, A., Gallagher, E. R., Admane, S., Jackson, V. A., . . Lynch, T. J. (2010). Early palliative care for patients with metastatic non-small-cell lung cancer. New England Journal of Medicine, 363(8), 733-742. http://www.nejm.org/doi/ pdf/10.1056/NEJMoa1000678

Warner, E., \& Gualtieri-Reed, T. (2014). Improving care for people with serious illness through innovative payerprovider partnerships. A palliative care toolkit and resource guide. New York: Center to Advance Palliative Care.

Wright, A. A., Keating, N. L., Balboni, T. A., Matulonis, U. A., Block, S. D., \& Prigerson, H. G. (2010). Place of death: Correlations with quality of life of patients with cancer and predictors of bereaved caregivers' mental health. Journal of Clinical Oncology, 28(29), 4457-4464. http://dx.doi.org/10.1200/JCO.2009.26.3863 
RTI International is an independent, nonprofit research organization dedicated to improving the human condition by turning knowledge into practice. RTI offers innovative research and technical solutions to governments and businesses worldwide in the areas of health and pharmaceuticals, education and training, surveys and statistics, advanced technology, international development, economic and social policy, energy and the environment, and laboratory and chemistry services.

The RTI Press complements traditional publication outlets by providing another way for RTI researchers to disseminate the knowledge they generate. This PDF document is offered as a public service of RTI International. 